\title{
Main causes of death in Dande, Angola: results from Verbal Autopsies of deaths occurring during 2009-2012
}

\author{
Edite Vila Nova Rosário ${ }^{1 *}$, Diogo Costa ${ }^{1,2}$, Luís Timóteo ${ }^{3}$, Ana Ambrósio Rodrigues ${ }^{4}$, Jorge Varanda,
} Susana Vaz Nery ${ }^{7 \dagger}$ and Miguel Brito ${ }^{1,8+}$

\begin{abstract}
Background: The Dande Health and Demographic Surveillance System (HDSS) located in Bengo Province, Angola, covers nearly 65,500 residents living in approximately 19,800 households. This study aims to describe the main causes of deaths (CoD) occurred within the HDSS, from 2009 to 2012, and to explore associations between demographic or socioeconomic factors and broad mortality groups (Group I-Communicable diseases, maternal, perinatal and nutritional conditions; Group II—Non-communicable diseases; Group III—Injuries; IND—Indeterminate).
\end{abstract}

Methods: Verbal Autopsies (VA) were performed after death identification during routine HDSS visits. Associations between broad groups of CoD and sex, age, education, socioeconomic position, place of residence and place of death, were explored using chi-square tests and fitting logistic regression models.

Results: From a total of 1488 deaths registered, 1009 verbal autopsies were performed and 798 of these were assigned a CoD based on the $10^{\text {th }}$ revision of the International Classification of Diseases (ICD-10).

Mortality was led by CD (61.0\%), followed by IND (18.3\%), NCD (11.6\%) and INJ (9.1\%). Intestinal infectious diseases, malnutrition and acute respiratory infections were the main contributors to under-five mortality (44.2 \%). Malaria was the most common CoD among children under 15 years old (38.6\%). Tuberculosis, traffic accidents and malaria led the CoD among adults aged 15-49 (13.5\%, $10.5 \%$ and $8.0 \%$ respectively). Among adults aged 50 or more, diseases of the circulatory system (23.2 \%) were the major CoD, followed by tuberculosis ( $8.2 \%$ ) and malaria (7.7\%). CD were more frequent CoD among less educated people (adjusted odds ratio, $95 \%$ confidence interval for none vs. 5 or more years of school: 1.68, 1.04-2.72).

Conclusion: Infectious diseases were the leading $\mathrm{CoD}$ in this region. Verbal autopsies proved useful to identify the main $\mathrm{CoD}$, being an important tool in settings where vital statistics are scarce and death registration systems have limitations.

Keywords: Verbal Autopsy, Angola, Health and demographic surveillance system, Causes of death

\section{Background}

Mortality data are essential for defining and evaluating public health policies and for inferring about the health status of the population in a country $[1,2]$. However, medical certification of cause of death is used in only one third of deaths occurring worldwide and a lack of accurate death registration systems is common in most

\footnotetext{
*Correspondence: edite.rosario@cisacaxito.org; cisa.evr@gmail.com ${ }^{\dagger}$ Equal contributors

${ }^{1}$ Health Research Centre of Angola (CISA), Caxito, Bengo, Angola Full list of author information is available at the end of the article
}

developing countries, particularly where mortality is highest [3].

As in other Sub-Sahara African countries, in Angola the causes of death and their determinants are not well documented [3-5]. In recent years, Angola faced great social and economic changes, resulting from the end of an armed conflict of almost 30 years. Furthermore, in less than a decade, a vibrant economy has transformed the country from a low income centrally-planned system to a middle income market economy [6, 7]. Such development brings changes in the societal and economic 
structure which, in turn, impacts health and mortality patterns [8-10]. However, the resources needed to implement an accurate and complete vital statistics system are not yet fulfilled.

Even though World Health Organization (WHO) provides estimates of deaths by cause for the country (the main causes in 2012 were respiratory infections, diarrhea, neonatal deaths, cardiovascular diseases and malaria [11]), these figures pertain to aggregated data, not describing regional patterns (for example, cause-specific mortality in identified malaria endemic areas). In Angola, death certification is only done routinely for violent deaths and on those occurring in hospitals or in other health facilities [12]. For example, it is estimated that only about $5 \%$ of under-five children deaths take place at the hospitals [13] and that about $45 \%$ of the population has access to any type of health care [14]. The system thus fails to collect data on deaths occurring at home or at small health units and is therefore insufficient to reliably inform about mortality.

In countries with limited or non-existent death registration systems, the use of verbal autopsy (VA) is recommended to ascertain probable causes of death [1]. Verbal autopsy as a method to estimate cause-specific mortality, but also to study risk factors for specific diseases and the effects of public health interventions, is increasing in the developing world and is now used in more than 115 countries [1, 3]. Verbal autopsy systems are often developed as part of population monitoring platforms, namely Health and Demographic Surveillance Systems (HDSS), to allow longitudinal assessment of mortality trends and exploration of associated factors. The Dande HDSS (Bengo, northern Angola), where a VA system is implemented since 2010, provides such an opportunity [15].

This study aims to describe the main causes of deaths and explore factors associated with broad mortality groups for the period 2009-2012 in the Dande HDSS study area.

\section{Methods}

\section{Study area and population}

The Dande HDSS is located in Dande municipality, Bengo province, about $60 \mathrm{~km}$ northeast of Luanda, in Northen Angola. It was established and is managed by CISA-Health Research Centre in Angola-to overcome the scarcity of complete vital records, the lack of knowledge on demographic characteristics and living conditions of the population in this region and to create a sampling frame for epidemiological studies. Detailed information about the HDSS scope, design and implementation, methodology and data management procedures is published elsewhere [15].

The Dande HDSS covers all 71 hamlets of Caxito, Mabubas and Úcua, 3 of the 6 communes composing the Dande municipality, with a contiguous total area of $4763.6 \mathrm{~km}^{2}$ (Fig. 1).

Thirty hamlets are considered urban (agglomerations of 2000 or more inhabitants and basic infrastructures) and 41 are rural, according to the National Institute of Statistics of Angola (INEA) definitions [16]. In the initial census, performed in 2009, a population of 59,683 individuals living in 15,643 households was registered. In December 2012, the population covered by the HDSS was of 60,614 residents.

The Angolan Health System encompasses public and private sector providers, the former being the main provider. The national public health system is hierarchically organized in primary health care centers (these include small health care units, nursing centres and municipal hospitals), secondary level general hospitals and tertiary level reference hospitals [14]. The Dande Municipality, where the study area is located, is served by eleven primary health care facilities, one health centre, one maternal and infant centre, one municipal hospital and one general hospital [17].

\section{Data collection}

After the initial census, that was performed between August 2009 and March 2010, 18 fieldworkers periodically visited all the households of the study area to record basic demographic events, including reported deaths (quarterly update rounds took place until the end of 2011 and, due to logistic and administrative issues, a single 6-month round was performed in 2012). This study covers the deaths which occurred from August 2009 to December 2012.

After a mourning period of approximately a month and a half, three fieldworkers specifically trained to apply the VA tool, individually visited the households of the deceased and interviewed the caregiver of the deceased or other close relative.

Three versions of the standard INDEPTH/WHO model of the VA questionnaire $[18,19]$ were used (one for neonatal deaths -0 to 27 days, one for children -28 days to 14 years, and one for adults-15 years or more) adapted to include the main pathologies known in the region and local terminologies. The VA questionnaires are structured into different sections, and include open questions on the circumstances of the death, sociodemographic data of the interviewee and of the deceased, a checklist of signs and symptoms experienced before death (including their duration), accidents, accessibility to health care, place of death and any available health records, namely vaccination register, laboratory exams results and death certificate.

\section{Assigning the cause of death}

To ascertain the probable cause of death, each questionnaire was interpreted independently by two physicians 

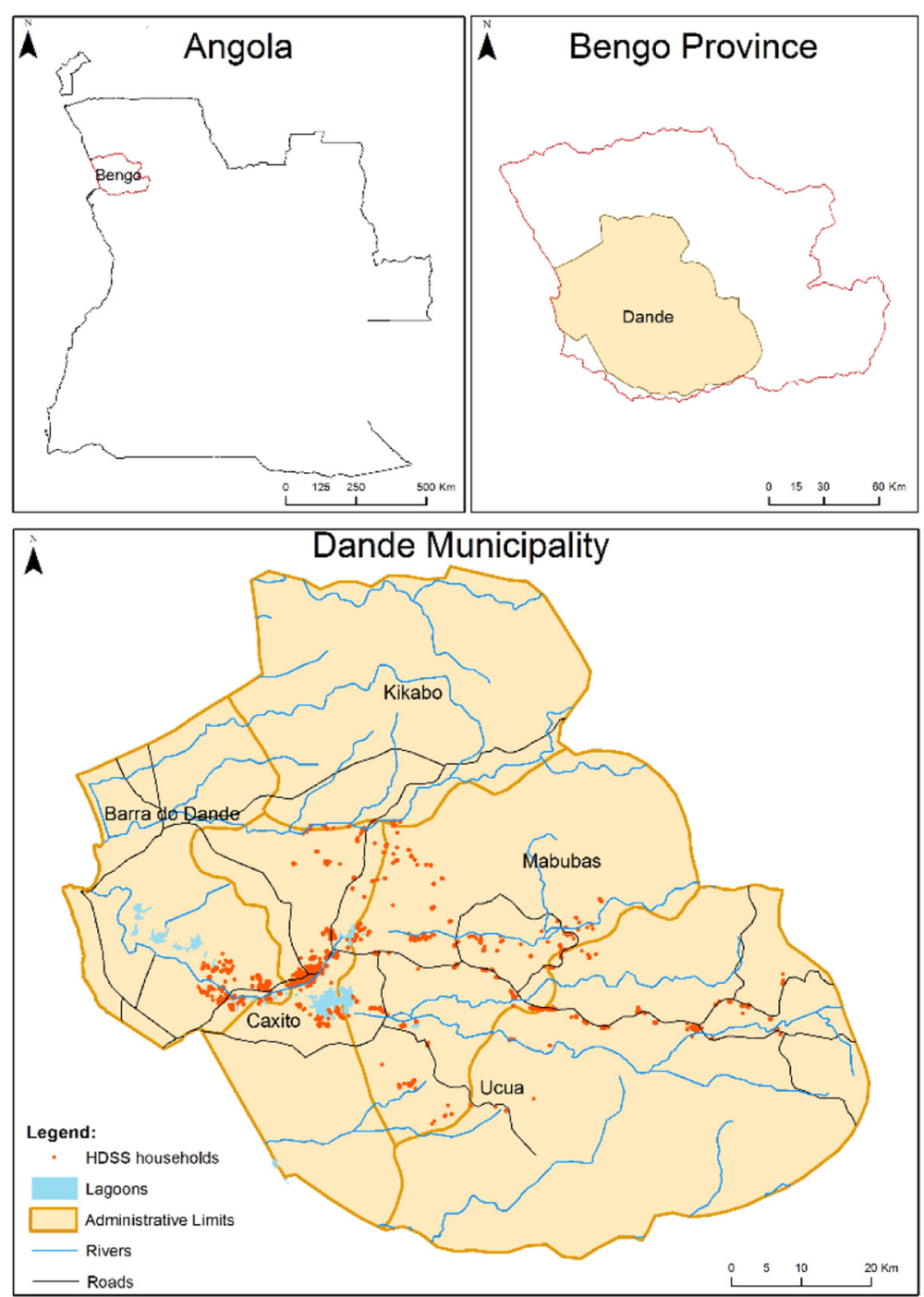

Fig. 1 Map of the study area_Angola, Bengo Province, Dande Municipality

with local experience and trained on reviewing the VA questionnaires and assigning the cause of death. Training of physicians in the interpretation of the questionnaires was based on case definition using clinical diagnostic algorithms adapted from other VA systems [18, 20-23]. In case of discordance between the two physicians, the questionnaire was reviewed by a third physician and the majority rule was applied. Otherwise, if the third physician determined a different cause of death, the case was registered as indeterminate $[24,25]$.

The reading physicians were staff of the General Provincial Hospital, partner of the Health Research Centre of Angola and also the setting where the Centre is located. This clinical staff is mostly composed of Angolan national practitioners, although there are also foreign doctors (Cuban) working in this context for several years, as part of bilateral cooperation agreements between governments. For each VA questionnaire reading, the Centre paid a symbolic fee to the physicians, in order to compensate their participation. The Hospital benefits of the results of the verbal autopsy system, an important tool to the knowledge of the main causes of death in the study area.

The international form for medical certificate of cause of death was used, which is divided in two parts: the first describes the sequence of morbid conditions directly leading to death; the second describes other diseases or conditions which may have contributed to the death, but which were not involved in the fatal sequence.

Though the international death certificate typically uses the underlying cause of death, ascertaining a single cause of death from various possible causes identifiable from the reported symptoms may be inappropriate using VA data [1]. Mortality is often due to the effects of 
multiple conditions, and particularly in the case of infant death, it is more difficult to distinguish, in terms of semiology and differential diagnosis, the main causes that lead to death. Additionally, concerning adults is quite straightforward the distinction between comorbidities and underlying cause for death. For those reasons, multiple causes of death were considered for children under 15 years old and the underlying cause of death was considered for adults.

Causes of death were coded using the $10^{\text {th }}$ revision of the International Classification of Diseases (ICD-10) [26].

\section{Categorization of causes of death}

After classification based on ICD-10, specific causes of death were categorized according to the three mortality groups of the Global Burden of Disease (GBD) structure [27] and a fourth for indeterminate causes.

Group I included deaths attributed to communicable diseases and maternal, perinatal and nutritional conditions (CD): intestinal infectious diseases, tuberculosis (TB), other bacterial diseases, rabies, measles, viral hepatitis, human immunodeficiency virus (HIV), malaria, nutritional deficiencies, meningitis, otitis, conditions related to or aggravated by the pregnancy, childbirth or by the puerperium (maternal causes or obstetric causes) and certain conditions originating in the perinatal period.

Group II consisted of non-communicable diseases (NCD): malignant neoplasms, respiratory disorders, mental and behavioral disorders, epilepsy, diseases of the circulatory system, diseases of the digestive system, diseases of the genitourinary system, skin disorders and congenital abnormalities.

Group III comprised of injuries (INJ): injury, poisoning and other consequences of external causes of morbidity and mortality, such as burns and corrosions, toxic effects of substances chiefly nonmedicinal as to source, complications of surgical and medical care, traffic accidents, intentional self-harm, assault, event of undetermined intent, accidental drowning and submersion, struck by thrown, projected or falling object, falls, contact with scorpions, exposure to unspecific electric current and handgun discharge.

Indeterminate causes (IND) included: unknown and unspecified causes of mortality (physicians assigned the VA as ill-defined or as unknown cause of mortality-codes R95-R99 of ICD-10) and those cases that the VA method didn't reach a consensus on the cause of death.

\section{Demographic and socioeconomic characteristics}

During the initial census of the HDSS, implemented from August 2009 to March 2010, and during the $5^{\text {th }}$ update round that took place from September to December 2011, demographic characteristics (age and sex) and information on household conditions were collected. In each HDSS update round, demographic events are updated and all the information is collected for new members in the study area.

The educational level of the deceased (or the mother's educational level for children under 15 years of age) was categorized in three groups: no formal education; from one to four-corresponding to primary education; and five or more years of completed schooling.

Several household conditions were used to create a proxy socioeconomic position (SEP) index: type of walls (made of clay, wattle and daub, bricks), type of roof (straw, tin, tile, other), main source of drinking water (public fountain, piped water, river, irrigation ditch, well, plumbed, public tank) and existence of a latrine (with or without water and private or shared with neighbors). For each identified death the most recently updated information on their household conditions was used. The place of residence of the deceased (dichotomized in rural or urban) and place of death (in a health facility or in other/unknown location), were also used.

\section{Statistical analysis}

Proportions of the main causes of deaths were computed by sex and the six age groups most commonly used to describe age-specific mortality $(<28$ days, from 28 days to 12 months, 1 to 5 years, 5 to 15 years, 15 to 50 years and 50 or more years of age).

The chi-square test was used to compare proportions of deaths with and without an assigned cause according to sex and age (Additional file 1).

Principal component analysis (PCA) was used to summarize household conditions and create the SEP index. The first principal component extracted, which explained $17.8 \%$ of variance was then categorized into SEP quintiles (lowest, low, medium, high and highest).

Proportions of the grouped causes of death (CD, NCD, INJ and IND), according to sex, age (for the bivariate and multivariate analysis, age was re-categorized in four groups: under 5 years, 5 to $14,15-49$ and 50 or more), educational level, the quintiles of the SEP index, place of residence (rural or urban), and place of death (in a health facility or other/unknown) were compared using the chi-square test.

The WHO standard population (2000-2025) [28] was used to compute standardized deaths rates using the direct method.

Fully adjusted multivariate logistic regression models were fitted and odds ratios (95\% confidence intervals OR, $95 \% \mathrm{CI}$ ) computed to estimate associations between each main group of cause of death (CD, NCD, INJ and IND) and all demographic and socioeconomic characteristics considered in the bivariate analysis. Models were additionally adjusted for multiple causes of death 
by adding a dichotomized dummy variable signaling multiple cause cases. Only participants with complete information were used in the regression models. Two variables presented missing information, namely education (5.5\% of missing values) and socio economic position index ( $0.1 \%$ missing). Analysis was performed using the software SPSS v.22.

\section{Ethical consideration}

In the Dande HDSS, we sought verbal consent from household heads or any other adult member of the household, to update the health and demographic information of household members every 6 months. Verbal autopsies for the reported deaths were included as operations of the Dande HDSS.

As a health and demographic surveillance system with frequent visits to households to update demographic information, verbal consent was deemed appropriate for monitoring of the demographic events. In the case of VA, informed written consent (signed or fingerprinted in case of illiteracy) was required before the conduct of each interview. The information given out to respondents during the consent process was documented and fieldworkers have been adequately trained to administer the consent. The consent information included the aim of the verbal autopsy system, the content of the participation of the caregiver (to answer to an interview, giving information about the death of her/his relative), the voluntary feature of participation and the confidentiality of the information. Consent forms were kept safe, strictly confidential and separated from questionnaires.

The scientific committee of CISA, composed of several biomedical scientists, epidemiology and public health experts acted as an institutional review board (IRB) for all studies and reviewed the procedures of the HDSS and VA systems. The IRB approved the consent process and also asked for the advice of the Ministry of Health and of Provincial Health Governor. According to their recommendations, the forms of the HDSS and VA were approved and registered in the National Statistics Institute of Angola (INEA).

The STROBE guidelines were followed.

\section{Results}

From August 2009 to December 2012 a total of 1488 deaths were registered by the Dande HDSS, 811 (54.5\%) were males. The mean age at death was 30.0 years (standard deviation $=28.1$ ), with $513(34.5 \%)$ occurring in children under 5 years of age.

The crude mortality rate in 2010 was 8.7 per 1000 inhabitants, (95 \% confidence interval CI: 8.0-9.5) and 8.1 per 1000 (95\% CI: 7.4-8.8) in 2011. In 2012, the estimated crude mortality rate was 6.6 (95\% CI: 5.9-7.2). The standardized deaths rates (WHO standard population 2000-2025) were 10.8, 9.8 and 9.3 per 1000 for 2010, 2011 and 2012 respectively.

Infant mortality rate was 67.1 deaths per 1000 live births in 2010, 78.4 in 2011, and 60.2 in 2012. Under five mortality rate was 90.9, 93.6 and 71.5 per 1000 live births in 2010, 2011 and 2012 respectively.

Of the 1488 deaths, 1009 (67.8\%) verbal autopsies were made. Five families refused $(0.3 \%)$ to participate in the study. The difference between the number of reported deaths and the number of interviews was mainly due to 316 families that migrated out of or in the study area (21.2\%), 27 individuals who lived alone at the time of death $(1.8 \%)$ making it very difficult to find a primary caregiver to interview and 131 families who were not at home in the various attempts done to conduct the interview $(8.8 \%)$. The periodic visits of the HDSS to households allows the surveillance of the population, however when individuals migrate within the study area they are registered in the new house only during the next update round, making it difficult to locate them in the periods between the visits.

Of the 1009 completed verbal autopsies 798 were reviewed and a cause of death was attributed. Of the total of 798 with assigned causes of death, 458 (57 \%) had to be reviewed by a third doctor. The remaining 211 were not included in the analysis because they were not read by at least two physicians and therefore had no cause of death assigned. When considering multiple causes of death in children, a total of 934 causes of death were assigned and included in the current analysis. A statistically significant difference in the proportion of deaths with and without a VA reviewed was observed considering the six-age group categories, and no sexdifference was found (Additional file 1: Table S1).

This study revealed that malaria is the most common cause of death in the Dande HDSS, Angola, for the period August 2009 to December 2012, affecting mostly children and adolescents less than 15 years of age. Intestinal infectious diseases, malnutrition and pneumonia were also main contributors to child mortality. For adults, the most common causes of death are diseases of the circulatory system, TB, traffic accidents and malaria.

\section{Specific cause of death by age group and sex Neonatal deaths}

All deaths in female infants $<28$ days and for $47.4 \%$ of males, were classified as having a perinatal condition as cause of death (Table 1).

\section{Children}

In children from 28 days to 11 months of age (Table 2), the main causes of death were intestinal infectious diseases (female: $26.7 \%$; male: $34.4 \%$ ) and malaria (female: $19.8 \%$; male: $26.7 \%$ ). Those were followed by 
Table 1 Causes of neonatal death (children $<28$ days)

\begin{tabular}{|c|c|c|c|c|c|c|}
\hline \multirow[t]{2}{*}{$<28$ days } & \multicolumn{2}{|c|}{ Female $(n=8)$} & \multicolumn{2}{|c|}{ Male $(n=19)$} & \multicolumn{2}{|c|}{ Total $(N=27)$} \\
\hline & $\mathrm{N}$ & $\%$ & $n$ & $\%$ & $\mathrm{~N}$ & $\%$ \\
\hline Perinatal period conditions & 8 & 100.0 & 9 & 47.4 & 17 & 63.0 \\
\hline Intestinal infectious diseases (diarrhea) & 0 & 0.0 & 1 & 5.3 & 1 & 3.7 \\
\hline Malaria & 0 & 0.0 & 1 & 5.3 & 1 & 3.7 \\
\hline Meningitis & 0 & 0.0 & 1 & 5.3 & 1 & 3.7 \\
\hline Traffic accidents & 0 & 0.0 & 1 & 5.3 & 1 & 3.7 \\
\hline Unknow (R95-R99) & 0 & 0.0 & 1 & 5.3 & 1 & 3.7 \\
\hline Indeterminate & 0 & 0.0 & 5 & 26.3 & 5 & 18.5 \\
\hline Total & 8 & & 19 & & 27 & \\
\hline
\end{tabular}

pneumonia (female: $18.6 \%$; male: $14.4 \%$ ) and malnutrition (female: $15.1 \%$; male: $11.1 \%$ ).

The same diseases were the main causes of deaths among children aged $1-4$ years (Table 3): malaria (female: $32.8 \%$; male: $35.4 \%$ ), intestinal infectious diseases (female: $17.6 \%$; male: $22.0 \%$ ), malnutrition (female: $16.0 \%$; male: $13.4 \%$ ) and pneumonia (female: $13.0 \%$; male: $6.3 \%$ ).

Malaria was also the leading cause of death among 514 years old children (Table 4), (female: $28.1 \%$; male: $38.2 \%$ ) followed by intestinal infectious diseases (female: $18.8 \%$; male: $17.6 \%$ ). The deaths due to accidental drowning and submersion (female: $3.1 \%$; male: $14.1 \%$ ) were the third specific cause of death for this age group.

\section{Adults}

In adults (Table 5), a quarter of all deaths were classified as indeterminate $(25.0 \%)$. In the age group $15-49$ years,
$11.3 \%$ of women died of causes related with pregnancy, childbirth and puerperium, followed by malaria and diseases of the circulatory system (both with $7.5 \%$ ). The main causes for male death were TB (15.8\%), traffic accidents $(13.3 \%)$ and malaria $(8.3 \%)$.

From age 50 onwards (Table 6), $30.0 \%$ of all deaths had indeterminate causes of death. Diseases of the circulatory system were the most common cause of death identified for both sexes (female: $27.6 \%$; male: $18.6 \%$ ), followed by TB (female: $6.7 \%$; male: $9.8 \%$ ) and malaria (female: $7.6 \%$; male: $7.8 \%$ ).

\section{Main categories of mortality according to demographic characteristics, socioeconomic indicators, place of residence and place of death}

When specific causes of death are aggregated into GBD main categories of mortality, $\mathrm{CD}$ are the leading cause of

Table 2 Causes of death among infants from 28 days to 11 months

\begin{tabular}{|c|c|c|c|c|c|c|}
\hline \multirow[t]{2}{*}{28 days- 11 months } & \multicolumn{2}{|c|}{ Female $(n=61)$} & \multicolumn{2}{|c|}{ Male $(n=63)$} & \multicolumn{2}{|c|}{ Total $(N=124)$} \\
\hline & $\mathrm{N}$ & $\%$ & $\mathrm{n}$ & $\%$ & N & $\%$ \\
\hline Intestinal infectious diseases (diarrhea) & 23 & 26.7 & 31 & 34.4 & 54 & 30.7 \\
\hline Malaria & 17 & 19.8 & 24 & 26.7 & 41 & 23.3 \\
\hline Acute respiratory infections (pneumonia) & 16 & 18.6 & 13 & 14.4 & 29 & 16.5 \\
\hline Malnutrition & 13 & 15.1 & 10 & 11.1 & 23 & 13.1 \\
\hline Meningitis & 4 & 4.7 & 1 & 1.1 & 5 & 2.8 \\
\hline Tuberculosis & 3 & 3.5 & 0 & 0.0 & 3 & 1.7 \\
\hline Anaemia & 3 & 3.5 & 0 & 0.0 & 3 & 1.7 \\
\hline Measles & 2 & 2.3 & 0 & 0.0 & 2 & 1.1 \\
\hline Hepatitis & 0 & 0.0 & 1 & 1.1 & 1 & 0.6 \\
\hline Lower respiratory infections & 0 & 0.0 & 1 & 1.1 & 1 & 0.6 \\
\hline Digestive system & 0 & 0.0 & 1 & 1.1 & 1 & 0.6 \\
\hline Perinatal period & 1 & 1.2 & 0 & 0.0 & 1 & 0.6 \\
\hline Burn and corrosions & 0 & 0.0 & 1 & 1.1 & 1 & 0.6 \\
\hline Accidental drowning and submersion & 0 & 0.0 & 1 & 1.1 & 1 & 0.6 \\
\hline Indeterminate & 4 & 4.7 & 6 & 6.7 & 10 & 5.7 \\
\hline Total (multiple causes considered) & 86 & & 90 & & 176 & \\
\hline
\end{tabular}


Table 3 Causes of death among children from 1 to 4 years of age

\begin{tabular}{|c|c|c|c|c|c|c|}
\hline \multirow[t]{2}{*}{$1-4$ years } & \multicolumn{2}{|c|}{ Female $(n=87)$} & \multicolumn{2}{|c|}{ Male $(n=95)$} & \multicolumn{2}{|c|}{ Total $(N=182)$} \\
\hline & $\mathrm{N}$ & $\%$ & $\mathrm{~N}$ & $\%$ & $\mathrm{~N}$ & $\%$ \\
\hline Malaria & 43 & 32.8 & 45 & 35.4 & 88 & 34.1 \\
\hline Intestinal infectious diseases (diarrhea) & 23 & 17.6 & 28 & 22.0 & 51 & 19.8 \\
\hline Malnutrition & 21 & 16.0 & 17 & 13.4 & 38 & 14.7 \\
\hline Acute respiratory infections (pneumonia) & 17 & 13.0 & 8 & 6.3 & 25 & 9.7 \\
\hline Measles & 5 & 3.8 & 7 & 5.5 & 12 & 4.6 \\
\hline Accidental drowning and submersion & 2 & 1.5 & 5 & 3.9 & 7 & 2.7 \\
\hline Burn and corrosion & 2 & 1.5 & 1 & 0.8 & 3 & 1.2 \\
\hline Infections of the skin & 1 & 0.8 & 2 & 1.6 & 3 & 1.2 \\
\hline Meningitis & 2 & 1.5 & 0 & 0.0 & 2 & 0.8 \\
\hline Congenital malform. circulatory system & 2 & 1.5 & 0 & 0.0 & 2 & 0.8 \\
\hline Sepsis & 1 & 0.8 & 1 & 0.8 & 2 & 0.8 \\
\hline HIV & 1 & 0.8 & 1 & 0.8 & 2 & 0.8 \\
\hline Anaemia & 0 & 0.0 & 2 & 1.6 & 2 & 0.8 \\
\hline Traffic accidents & 1 & 0.8 & 0 & 0.0 & 1 & 0.4 \\
\hline Falls & 1 & 0.8 & 0 & 0.0 & 1 & 0.4 \\
\hline Struck by thrown. proj. or falling object & 1 & 0.8 & 0 & 0.0 & 1 & 0.4 \\
\hline Digestive system & 0 & 0.0 & 1 & 0.8 & 1 & 0.4 \\
\hline Nephrotic syndrome & 0 & 0.0 & 1 & 0.8 & 1 & 0.4 \\
\hline Unknow (R95-R99) & 2 & 1.5 & 1 & 0.8 & 3 & 1.2 \\
\hline Indeterminate & 6 & 4.6 & 7 & 5.5 & 13 & 5.0 \\
\hline Total (multiple causes considered) & 131 & & 127 & & 258 & \\
\hline
\end{tabular}

deaths (61.0\%), followed by NCD (11.6\%) and INJ (9.1\%). Within CD deaths, $11.2 \%$ were attributed to nutritional conditions, $3.2 \%$ to perinatal conditions and $1.6 \%$ to maternal causes (Additional file 1: Table S2).

A total of $18.3 \%$ of the causes of death were IND (physicians assigned the VA as ill-defined or the three different physicians didn't reach a consensus on the suggested cause of death) (Table 7).

Statistically significant differences were found across all groups for age, education, living in an urban or rural setting and place of death. Deaths in all categories were proportionally more frequent in males than in females except for NCD, although no statistically significant sexdifference was found. Deaths from CD were more frequent in children under 5 years of age (causing $70.0 \%$ of deaths in this age group) while those due to NCD were more frequent in people older than 50 years $(63.0 \%)$. INJ were more frequent in the age group 15-49 years (causing $50.6 \%$ of deaths in this age group) and IND causes of death among adults 50 or more years $(40.4 \%)$. Mortality was highest among those with no formal education, except for the group of deaths attributed to INJ where people with 5 or more years of school died more frequently $(40.5 \%)$. There was no significant relation between the SEP index and the main groups of causes of death $(p=0.053)$. The majority of deaths with an attributed cause occurred in individuals living in urban areas $(p=<0.001)$.

Deaths due to $\mathrm{CD}$ or NCD were more frequent at health facilities (CD: $59.3 \%$ and NCD: $54.6 \%$ ) and deaths caused by INJ and IND occurred mostly on other/ unknown place (INJ: 74.1\%, IND: $58.5 \%$, respectively).

In general, the bivariate associations described above were confirmed in the fully adjusted regression models (Table 8). Children under five were more likely to die from $\mathrm{CD}$ when compared to adults aged 50 or more years (adjusted OR, 95 \% CI: 8.45, 5.33-13.41), and less likely to die from NCD (aOR $0.0995 \%$ CI 0.04-0.18). The odds of dying of injuries were 3.91 times $(95 \% \mathrm{CI}$ : 1.33-11.48) higher for children aged 5-14 years compared to adults older than 50 years and the number of IND causes of death tended to increase with age, independently of the remaining factors.

Compared to individuals with 5 or more years of education, those with no formal education were more likely to die of CD causes (aOR $1.6895 \%$ CI 1.04-2.72) and less likely to die of INJ ( $\mathrm{aOR} 0.4695 \% 0.21-0.98$ ), independently of age, sex, place of residence and place of death. When stratified by age (under 15 years of age or above), adults with no formal education were 
Table 4 Causes of death among children from 5 to 14 years of age

\begin{tabular}{|c|c|c|c|c|c|c|}
\hline \multirow[t]{2}{*}{$5-14$ years } & \multicolumn{2}{|c|}{ Female $(n=27)$} & \multicolumn{2}{|c|}{ Male $(n=31)$} & \multicolumn{2}{|c|}{ Total $(N=58)$} \\
\hline & N & $\%$ & N & $\%$ & N & $\%$ \\
\hline Malaria & 9 & 28.1 & 13 & 38.2 & 22 & 38.6 \\
\hline Intestinal infectious diseases (diarrhea) & 6 & 18.8 & 6 & 17.6 & 12 & 18.2 \\
\hline Accidental drowning and submersion & 1 & 3.1 & 5 & 14.7 & 6 & 9.1 \\
\hline Malnutrition & 1 & 3.1 & 2 & 5.9 & 3 & 4.5 \\
\hline Acute respiratory infections (pneumonia) & 2 & 6.3 & 0 & 0.0 & 2 & 3.0 \\
\hline Traffic accidents & 2 & 6.3 & 0 & 0.0 & 2 & 3.0 \\
\hline Tuberculosis & 0 & 0.0 & 1 & 2.9 & 1 & 1.5 \\
\hline Sepsis & 1 & 3.1 & 0 & 0.0 & 1 & 1.5 \\
\hline Rabies & 1 & 3.1 & 0 & 0.0 & 1 & 1.5 \\
\hline Otitis & 0 & 0.0 & 1 & 2.9 & 1 & 1.5 \\
\hline Injuries knee and lower leg & 1 & 3.1 & 0 & 0.0 & 1 & 1.5 \\
\hline Toxic effects of nonmedicinal substances & 0 & 0.0 & 1 & 2.9 & 1 & 1.5 \\
\hline Falls & 1 & 3.1 & 0 & 0.0 & 1 & 1.5 \\
\hline Exposure to unspecified electric current & 0 & 0.0 & 1 & 2.9 & 1 & 1.5 \\
\hline Meningitis & 1 & 3.1 & 0 & 0.0 & 1 & 1.5 \\
\hline Malign neoplasms & 1 & 3.1 & 0 & 0.0 & 1 & 1.5 \\
\hline Unknow (R95-R99) & 1 & 3.1 & 2 & 5.9 & 3 & 4.5 \\
\hline Indeterminate & 4 & 12.5 & 2 & 5.9 & 6 & 9.1 \\
\hline Total (multiple causes considered) & 32 & & 34 & & 66 & \\
\hline
\end{tabular}

significantly more likely to die of NCD causes (aOR 2.56 $95 \%$ CI 1.30-5.02) and significantly less likely to die of INJ (aOR $0.2995 \%$ CI 0.12-0.67) compared to those with 5 or more years of education (Fig. $2 f$ and g, respectively).

Compared to those in the highest quintile of the SEP index, individuals in the medium SEP index quintile were more likely to die from INJ (aOR $2.3195 \% \mathrm{CI}$ 1.00-5.30), with no other statistically significant association found for the remaining SEP groups.

Living in a rural setting represented 3.23 (95 \% CI: 1.71-6.10) higher odds of dying of INJ compared to residents in urban areas.

Deaths from CD and NCD, were more frequently observed in a health facility (aOR $0.5695 \%$ CI0.40-0.79 and $0.59,0.37-0.93$, respectively) and deaths from INJ were, expectedly, more frequent in other/unknown places (aOR $3.5795 \%$ CI 2.04-6.25).

\section{Discussion}

The high proportion of deaths occurring in children under five (33.9 \% of all deaths occur in this age group) is characteristic of a developing country $[4,29,30]$ and is consistent with the WHO latest mortality data estimates for Angola [11]. The 2012 WHO estimates for the distribution of causes of death in children under 5 , place respiratory diseases (17.1\%), diarrhea (14.6\%) and malaria (12.6\%) as the main causes of death, which is similar to our findings, although with a higher burden for deaths caused by malaria (28.2 \%), diarrhea (23.0\%) and a lower frequency of respiratory diseases as causes of death (11.9\%). Being the Dande HDSS study area a malaria endemic region $[31,32]$, there might be a tendency to over diagnose malaria and misclassify other fever deaths [33-35]. Over diagnosis of a particular cause of death, known as endemic in a particular region, has been pointed as one of the limitations of the VA methodology, reducing its performance [1, 4, 35]. One other factor that may have contributed to an overestimation of malaria and intestinal infections as causes of death, was the methodology we opted for assigning multiple causes for neonatal and children deaths. Multiple effects and several medical conditions influence mortality, particularly in children and the elderly [36], and in contexts like Dande, the selection of the underlying cause of death is often arbitrary [4, 37]. One of the arguments commonly used in favor of the methodology which involves assigning multiple causes of death is related with the lack of criteria to select a single cause of death to the detriment of others. If the VA system aims to understand epidemiological needs at a population level, rather than describe the causes of deaths at individual level, selecting a single cause may be less appropriate than assigning multiple 
Table 5 Causes of death among adults aged 15-49 years

\begin{tabular}{|c|c|c|c|c|c|c|}
\hline \multirow[t]{2}{*}{$15-49$ years } & \multicolumn{2}{|c|}{ Female } & \multicolumn{2}{|c|}{ Male } & \multicolumn{2}{|c|}{ Total } \\
\hline & $\mathrm{n}$ & $\%$ & N & $\%$ & N & $\%$ \\
\hline Tuberculosis & 8 & 10.0 & 19 & 15.8 & 27 & 13.5 \\
\hline Traffic accidents & 5 & 6.3 & 16 & 13.3 & 21 & 10.5 \\
\hline Malaria & 6 & 7.5 & 10 & 8.3 & 16 & 8.0 \\
\hline Circulatory system diseases & 6 & 7.5 & 5 & 4.2 & 11 & 5.5 \\
\hline Digestive system & 5 & 6.3 & 4 & 3.3 & 9 & 4.5 \\
\hline Preg. childbirth and puerperium & 9 & 11.3 & 0 & 0.0 & 9 & 4.5 \\
\hline Intestinal infectious diseases (diarrhea) & 3 & 3.8 & 4 & 3.3 & 7 & 3.5 \\
\hline HIV & 4 & 5.0 & 3 & 2.5 & 7 & 3.5 \\
\hline Accidental drowning and sub. & 3 & 3.8 & 4 & 3.3 & 7 & 3.5 \\
\hline Intentional self-harm & 0 & 0.0 & 4 & 3.3 & 4 & 2.0 \\
\hline Burn and corrosion & 2 & 2.5 & 1 & 0.8 & 3 & 1.5 \\
\hline Malignant neoplasms & 0 & 0.0 & 2 & 1.7 & 2 & 1.0 \\
\hline Epilepsy & 0 & 0.0 & 2 & 1.7 & 2 & 1.0 \\
\hline Acute respiratory infections (pneumonia) & 0 & 0.0 & 2 & 1.7 & 2 & 1.0 \\
\hline Unsp. electric current exposure & 0 & 0.0 & 2 & 1.7 & 2 & 1.0 \\
\hline Rabies & 1 & 1.3 & 0 & 0.0 & 1 & 0.5 \\
\hline Acute upper respir. Infections & 0 & 0.0 & 1 & 0.8 & 1 & 0.5 \\
\hline Conditions of lower respiratory tract & 0 & 0.0 & 1 & 0.8 & 1 & 0.5 \\
\hline Infections of the skin and subcut. Tissue & 0 & 0.0 & 1 & 0.8 & 1 & 0.5 \\
\hline Toxic effects of nonmedicinal substances & 1 & 1.3 & 0 & 0.0 & 1 & 0.5 \\
\hline Complications of surgical and medical care & 1 & 1.3 & 0 & 0.0 & 1 & 0.5 \\
\hline Handgun discharge & 0 & 0.0 & 1 & 0.8 & 1 & 0.5 \\
\hline Contact with scorpions & 0 & 0.0 & 1 & 0.8 & 1 & 0.5 \\
\hline Assault & 0 & 0.0 & 1 & 0.8 & 1 & 0.5 \\
\hline Event of undetermined intent & 0 & 0.0 & 1 & 0.8 & 1 & 0.5 \\
\hline Unknow (R95-R99) & 5 & 6.3 & 6 & 5.0 & 11 & 5.5 \\
\hline Indeterminate & 21 & 26.3 & 29 & 24.2 & 50 & 25.0 \\
\hline Total & 80 & & 120 & & 200 & \\
\hline
\end{tabular}

causes, particularly for the purposes of public-health support [1].

The most common causes of death among all adults aged 15-49 years old were TB and traffic accidents, although for women, pregnancy, childbirth and puerperium complications were responsible for the highest proportion of deaths. The National Plan for Development of Health identifies TB and traffic accidents as enormous problems for the population aged 15-45 years old [14], although accurate statistics are not provided. The Global Health Estimates [11], point to a lower proportion of TB and a higher burden of HIV deaths in this age group. As reported in other studies [4, 29], VA might have contributed to an underestimation of HIV-related deaths, since the co-morbidity of TB and HIV [38, 39] might have influenced the weight of both diagnoses.
Traffic accidents were recently pointed as the second major cause of death in the country by the government in the media [40]. The increased availability of motor vehicles and the improvement in life conditions is likely to raise the burden of traffic INJ in this age group [41], which adds to the poor road infrastructures and drinking and driving culture.

Regarding deaths among women of reproductive age, our results confirm the need to prioritize interventions to improve reproductive and maternal health, since maternal-related deaths rates in Angola are amongst the highest in the world [42], and the reduction of its burden is a specific goal envisaged in the Millennium Development Goals.

Among the eldest groups (50 or more years of age), circulatory system diseases were the leading causes of 
Table 6 Causes of death among adults aged 50 or more years

\begin{tabular}{|c|c|c|c|c|c|c|}
\hline \multirow[t]{2}{*}{50 years +} & \multicolumn{2}{|c|}{ Female } & \multicolumn{2}{|c|}{ Male } & \multicolumn{2}{|c|}{ Total } \\
\hline & $\mathrm{N}$ & $\%$ & $n$ & $\%$ & $\mathrm{~N}$ & $\%$ \\
\hline Circulatory system diseases & 29 & 27.6 & 19 & 18.6 & 48 & 23.2 \\
\hline Tuberculosis & 7 & 6.7 & 10 & 9.8 & 17 & 8.2 \\
\hline Malaria & 8 & 7.6 & 8 & 7.8 & 16 & 7.7 \\
\hline Intestinal infectious diseases (diarrhea) & 3 & 2.9 & 6 & 5.9 & 9 & 4.3 \\
\hline Malignant neoplasms & 5 & 4.8 & 3 & 2.9 & 8 & 3.9 \\
\hline Traffic accidents & 2 & 1.9 & 5 & 4.9 & 7 & 3.4 \\
\hline Acute respiratory infections (pneumonia) & 4 & 3.8 & 3 & 2.9 & 7 & 3.4 \\
\hline HIV & 4 & 3.8 & 1 & 1.0 & 5 & 2.4 \\
\hline Diabetes Mellitus & 1 & 1.0 & 4 & 3.9 & 5 & 2.4 \\
\hline Digestive system & 1 & 1.0 & 3 & 2.9 & 4 & 1.9 \\
\hline Intentional self-harm & 2 & 1.9 & 1 & 1.0 & 3 & 1.4 \\
\hline Accidental drowning and sub. & 0 & 0.0 & 2 & 2.0 & 2 & 1.0 \\
\hline Rabies & 1 & 1.0 & 0 & 0.0 & 1 & 0.5 \\
\hline Mental and behavioural disorders & 0 & 0.0 & 1 & 1.0 & 1 & 0.5 \\
\hline Infections of the skin and subcutaneous tissue & 0 & 0.0 & 1 & 1.0 & 1 & 0.5 \\
\hline Injury of unspecified body region & 1 & 1.0 & 0 & 0.0 & 1 & 0.5 \\
\hline Exposure to unspecified electric current & 1 & 1.0 & 0 & 0.0 & 1 & 0.5 \\
\hline Sepsis & 0 & 0.0 & 1 & 1.0 & 1 & 0.5 \\
\hline Asthma & 0 & 0.0 & 1 & 1.0 & 1 & 0.5 \\
\hline Unknow (R95-R99) & 1 & 1.0 & 6 & 5.9 & 7 & 3.4 \\
\hline Indeterminate & 35 & 33.3 & 27 & 26.5 & 62 & 30.0 \\
\hline Total & 105 & & 102 & & 207 & \\
\hline
\end{tabular}

death. Such finding is consistent with the results from other studies showing that deaths related to the circulatory system, particularly cardiovascular diseases, increases with age $[5,29,33,43]$. In 2010, our group conducted a community-based survey of 1464 adults residing in the Dande municipality, and found a $23 \%$ prevalence of hypertension [44]. These findings suggest an expected increase in the burden attributable to chronic diseases among adults, resultant from the country's fast economic growth and lifestyles changes.

Overall, our results show similar distributions of deaths by broad GBD groups compared to those found in Tanzania [33], particularly in what concerns NCD (12\% in our study vs. $15 \%$ in Tanzania) and INJ ( $9 \%$ in both studies). The higher estimate of $\mathrm{CD}$ found in our study (61\%) might be explained by the inclusion of children (the Tanzania study, focused on adult deaths, and found $41 \%$ of CD deaths) and is more close to the findings of a study among adolescents and adults conducted in rural western Kenya, which found $74 \%$ of deaths caused by CD [29].

Mortality in these regions is still largely marked by infectious diseases, despite the increasing deaths caused by
NCD and INJ. This phenomenon, which a majority of developing countries in Africa is facing, has been called the "triple burden" $[29,45]$. Some of the factors usually linked to the increasing prevalence of NCD and INJ in developing countries are related with socioeconomic conditions improvement which impacts lifestyles changes. Therefore we explored the associations of socioeconomic conditions in broad mortality groups. For this, we used essentially two SEP proxy measures: the educational level and the quintiles of an index summarizing household conditions, which has been extensively used in other HDSSs to study inequalities in several health outcomes $[8,25,29,33,46-48]$.

No significant associations were observed according to the quintiles of the SEP index created, although point estimates suggest positive associations in those groups who might be considered socially disadvantaged, compared to those less disadvantaged, for CD and NCD. The gradient observed in $\mathrm{CD}$ deaths according to the educational level, is in line with the results of a recent study conducted in Chakaria, Bangladesh, where mortality due to $C D$ was more frequent among the poorest [46]. In the same study, NCD as leading causes of death also 
Table 7 Distribution of the main causes of death according to demographic and socioeconomic characteristics

\begin{tabular}{|c|c|c|c|c|c|c|c|}
\hline & & Total & Communicable & Non-Communicable & Injuries & Indeterminate & \\
\hline & & $n$ & n (\%) & n (\%) & n (\%) & n (\%) & $p$ \\
\hline \multirow[t]{2}{*}{ Sex } & Female & 442 & $278(48.8)$ & $55(50.9)$ & $30(35.3)$ & $79(46.2)$ & 0.109 \\
\hline & Male & 492 & $292(51.2)$ & $53(49.1)$ & $55(64.7)$ & $92(53.8)$ & \\
\hline \multirow[t]{4}{*}{ Age (years) } & $<5$ & 461 & $399(70.0)$ & $14(13.0)$ & $16(18.8)$ & $32(18.7)$ & $<0.001$ \\
\hline & $5-14$ & 66 & $44(7.7)$ & $1(0.9)$ & $12(14.1)$ & $9(5.3)$ & \\
\hline & $15-49$ & 200 & $71(12.5)$ & $25(23.1)$ & $43(50.6)$ & $61(35.7)$ & \\
\hline & $50+$ & 207 & $56(9.8)$ & $68(63.0)$ & $14(16.5)$ & $69(40.4)$ & \\
\hline \multirow[t]{3}{*}{ Education ${ }^{\mathrm{a}}$ (years) } & None & 332 & $202(38.1)$ & $52(49.1)$ & $16(20.3)$ & $62(36.9)$ & 0.001 \\
\hline & 1 to 4 & 316 & $202(38.1)$ & $27(25.5)$ & $31(39.2)$ & $56(33.3)$ & \\
\hline & 5 or more & 235 & $126(23.8)$ & $27(25.5)$ & $32(40.5)$ & $50(29.8)$ & \\
\hline \multirow[t]{5}{*}{ SEP index } & Lowest & 186 & $121(21.2)$ & $23(21.3)$ & $12(14.1)$ & $30(17.6)$ & 0.053 \\
\hline & Low & 222 & $143(25.1)$ & $27(25.0)$ & $13(15.3)$ & 39 (22.9) & \\
\hline & Medium & 170 & $108(18.9)$ & $14(13.0)$ & $21(24.7)$ & $27(15.9)$ & \\
\hline & High & 171 & $107(18.8)$ & $18(16.7)$ & $16(18.7)$ & $30(17.6)$ & \\
\hline & Highest & 184 & $91(16.0)$ & $26(24.1)$ & $23(27.1)$ & $44(25.9)$ & \\
\hline \multirow[t]{2}{*}{ Residence } & Rural & 180 & $88(15.4)$ & $23(21.3)$ & $32(37.6)$ & 37 (21.6) & $<0.001$ \\
\hline & Urban & 754 & 482 (84.6) & 85 (78.7) & $53(62.4)$ & $134(78.4)$ & \\
\hline \multirow[t]{2}{*}{ Place of death } & Health facility & 490 & 338 (59.3) & $59(54.6)$ & $22(25.9)$ & $71(41.5)$ & $<0.001$ \\
\hline & Other/unknown & 444 & $232(40.7)$ & $49(45.4)$ & $63(74.1)$ & $100(58.5)$ & \\
\hline
\end{tabular}

$p$ p-value-chi square test

SEP index: socioeconomic position index

${ }^{a}$ For participants under 15 years of age, the mother's educational level was considered

Table 8 Associations between causes of death and socioeconomic characteristics

\begin{tabular}{|c|c|c|c|c|c|}
\hline & & Communicable & Non- Communicable & Injuries & Indeterminate \\
\hline & & *AOR (95 \% Cl) & *AOR (95 \% Cl) & *AOR (95 \% Cl) & ${ }^{*} A O R(95 \% \mathrm{Cl})$ \\
\hline \multirow[t]{2}{*}{ Sex } & Male & 1 & 1 & 1 & 1 \\
\hline & Female & $0.92(0.64-1.32)$ & $1.11(0.67-1.82)$ & $0.77(0.44-1.37)$ & $1.18(0.79-1.76)$ \\
\hline \multirow[t]{4}{*}{ Age (years) } & $<5$ & $8.45(5.33-13.41)$ & $0.09(0.04-0.18)$ & $1.01(0.45-2.26)$ & $0.33(0.20-0.55)$ \\
\hline & $5-14$ & $5.36(2.49-11.54)$ & n.a. & $3.91(1.33-11.48)$ & $0.38(0.15-0.97)$ \\
\hline & $15-49$ & $1.67(1.05-2.64)$ & $0.29(0.16-0.50)$ & $3.60(1.78-7.29)$ & $0.85(0.54-1.33)$ \\
\hline & $50+$ & 1 & 1 & 1 & 1 \\
\hline \multirow[t]{3}{*}{ Education ${ }^{\text {a }}$ (years) } & None & $1.68(1.04-2.72)$ & $1.08(0.57-2.07)$ & $0.46(0.21-0.98)$ & $0.77(0.46-1.30)$ \\
\hline & $1-4$ & $1.15(0.74-1.79)$ & $0.81(0.44-1.51)$ & $0.93(0.50-1.75)$ & $1.03(0.64-1.65)$ \\
\hline & 5 or more & 1 & 1 & 1 & 1 \\
\hline \multirow[t]{5}{*}{ SEP index } & Lowest & $1.16(0.66-2.05)$ & $1.04(0.50-2.16)$ & $1.18(0.48-2.92)$ & $0.73(0.40-1.34)$ \\
\hline & Low & $1.21(0.71-2.05)$ & $0.96(0.49-1.88)$ & $0.86(0.37-1.98)$ & $0.84(0.49-1.46)$ \\
\hline & Med & $0.84(0.47-1.50)$ & $0.80(0.36-1.77)$ & $2.31(1.00-5.30)$ & $0.84(0.46-1.56)$ \\
\hline & High & $0.81(0.46-1.44)$ & $0.96(0.46-2.01)$ & $1.43(0.63-3.28)$ & $1.03(0.57-1.86)$ \\
\hline & Highest & 1 & 1 & 1 & 1 \\
\hline \multirow[t]{2}{*}{ Residence } & Rural & $0.65(0.41-1.05)$ & $0.86(0.47-1.60)$ & $3.23(1.71-6.10)$ & $0.87(0.53-1.42)$ \\
\hline & Urban & 1 & 1 & 1 & 1 \\
\hline \multirow[t]{2}{*}{ Place of death } & Health facility & 1 & 1 & 1 & 1 \\
\hline & Other/unknown & $0.56(0.40-0.79)$ & $0.59(0.37-0.93)$ & $3.57(2.04-6.25)$ & $1.44(0.99-2.08)$ \\
\hline
\end{tabular}

*AOR $(95 \% \mathrm{Cl})=$ adjusted odds ratio (95\% confidence Interval). adjusted for all variables listed and for multiple causes SEP index: socioeconomic position index

${ }^{a}$ For participants under 15 years of age, the mother's educational level was considered 
a Communicable Diseases

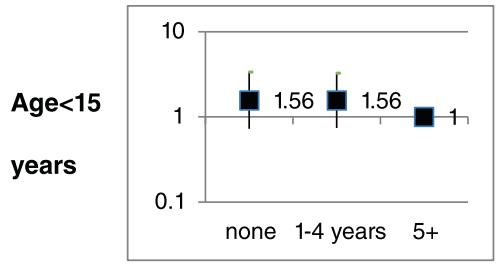

e

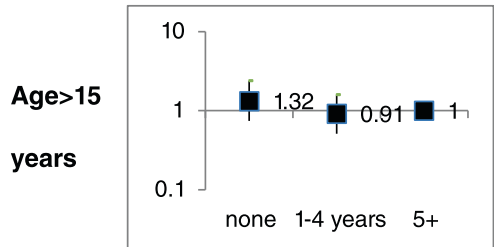

b

Non-Communicable Diseases

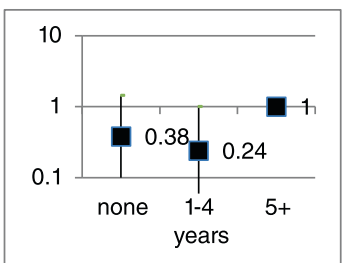

f

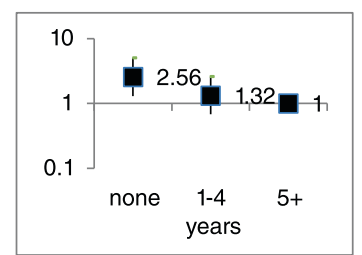

C

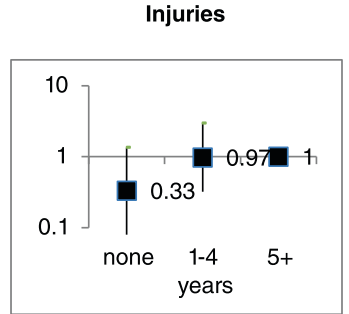

g

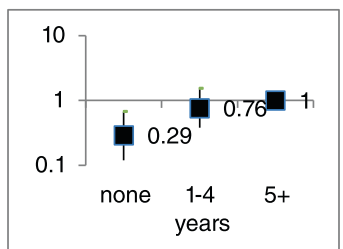

d

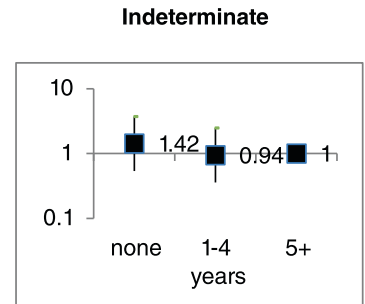

h

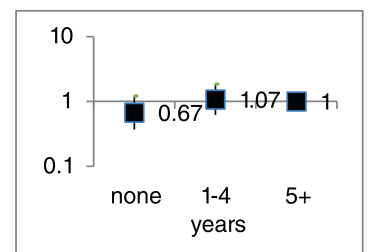

Fig. 2 Associations (adjusted Odds Ratio, OR, 95\% Confidence Intervals, Cl) between Educational level (none, 1-4 years, 5 or more years of schooling) and main causes of death among those under 15 years of age and among those aged 15 or more for deaths caused by Communicable Diseases (a), Non-Communicable Diseases (b), Injuries (c), and Indeterminate (d). Marks represent OR estimates and bars represent $95 \% \mathrm{Cl}$. Models for under-15 were adjusted for sex, place of residence, local of death and multiple causes and models for 15 years or more were adjusted for sex, place of residence and local of death

concentrated among the population from the higher SEP groups [47], contrasting our results for adult NCD deaths according to the educational level as shown in Fig. 2f. This may suggest that the expected epidemiological transition in the leading causes of death is only in its early stage $[49,50]$.

Nevertheless, we would expect a steeper socioeconomic gradient for all main causes of death, given the fast development observed in the country in the last decade [6] and the known inequalities in mortality observed worldwide and in the country [42]. It would be interesting to explore other SEP measures, such as the subjective social status [51], potentially more useful to disentangle underlying inequalities of health in this context.

Most deaths studied occurred in the urban area, following the population distribution in the study area. The higher likelihood of an INJ death in residents from rural compared to urban hamlets was a rather surprising finding. However, we should notice that only the deceased residence is known, and death might have happened in an urban area (outside a health facility). When isolating the specific causes of death by injuries, we can observe the weight of those related with traffic accidents and accidental drowning and submersion, both in rural and urban areas. In rural areas the villages are situated along the road and those have relatively poor conditions and some traffic, especially of trucks transporting raw materials. This has been the cause of road and pedestrian accidents. Likewise, people living in rural areas have to travel long distances which may explain the higher likelihood to die of INJ, particularly of transport accidents.

To better understand the context of INJ deaths, we recently included a specific module in our VA questionnaire, which will be the focus of future exploration (as the characterization and context of the main deaths caused by INJ in order to sustain public health interventions and specific campaigns).

Regarding place of death, CD and NCD deaths happened more commonly in a health facility. Nevertheless, more than $40 \%$ of $\mathrm{CD}$ and NCD deaths were registered outside a health facility, which emphasizes the usefulness of the VA system in the region.

The identification of causes of deaths through VA methodology in the scope of a continuous surveillance system is the strength of this study considering the lack of information in this context. In Angola where data on mortality is based on hospital and government reports, only the number of people dying under medical care is accounted for and death certificates lack accuracy [12]. However it is of crucial importance to know the causes associated to all deaths $[52,53]$. Bengo Province, where the study area is located, is reported as an endemic region for several infectious diseases responsible for morbidity and mortality in Angola [14, 31], however there is a very limited capacity of the health structures of registering and measuring the impact of that endemicity on mortality.

The estimated crude mortality for the 3 years encompassing the study period, suggests a decrease in rates, which is in line with the WHO estimates for the same period [11]. However, the estimated crude mortality rate 
for 2012 that we observed (6.6 per 1000, 95 \% CI: 5.9-7.2) may be underestimated: during this period, only one update HDSS round was performed, as opposed to the usual biannual rounds, thus fewer events were reported explaining the observed decrease.

In our study, $18.3 \%$ of deaths were IND, $8.7 \%$ for child and $27.5 \%$ for all adult deaths. This figure lies between the results found in other sub-saharan African countries, namely in Ethiopia (reporting VA deaths for the period 2009-2013) [5] where $14.9 \%$ of deaths were IND and a 2012 study from Tanzania reporting $31 \%$ deaths with indeterminate causes [33]. The high proportion of IND causes of death is a common limitation attributed to the VA system, that may result from the relatively low specificity and sensitivity of the VA tool for detecting some causes of death [4, 29], and from other influencing factors such as the interaction between interviewer and respondent, the setting where the VA is performed, recall bias and the subjectivity inherent to the physician review [1]. Further investigation of what determines indeterminate causes is warranted.

One other problem felt in the Dande HDSS, is the scarcity of physicians available to be trained to read VA questionnaires. In the future, the validity of the causes of death derived from VA reviewed by physicians should be tested with the use of a probabilistic approach, such as the InterVA method [1].

\section{Conclusions}

The findings of this study confirm international estimates on the main causes of death and provide a more detailed picture of mortality in this region, informing health policies and focused interventions. The VA system proves to be a useful tool in this context, where almost half of deaths occur outside a health facility and mortality data is scarce and often inaccurate.

Preventive measures should be given priority, since infectious diseases remain the major cause of death, despite the country's development in the past decade. Simultaneously, efforts tackling NCD and INJ cannot be neglected and should initiate immediately in order to prevent escalation of its burden.

\section{Additional file}

Additional file 1: Table S1. Proportion of deaths with and without a Verbal autopsy reviewed. Table S2 Distribution death attributable do communicable diseases according to demographic and socioeconomic characteristics. (DOCX $17 \mathrm{~kb}$ )

\section{Abbreviations}

CD, communicable diseases; CISA, Health Research Centre of Angola; CoD, causes of deaths; GBD, Global Burden of Disease; HDSS, health and demographic surveillance system; HIV, imunodeficiency virus; ICD-10, 10th revision of the
International Classification of Diseases; IND, indeterminate; INEA, National Institute of Statistics of Angola; INJ, injuries; IRB, institutional review board; NCD, Noncommunicable diseases; PCA, principal component analysis; SEP, socioeconomic position; TB, tuberculosis; VA, verbal autopsies; WHO, World Health Organization

\section{Acknowledgments}

We gratefully acknowledge the HDSS community, all staff involved in the VA system, the contribution of physicians and of Professor Maciel Barbosa for his valuable input.

\section{Fundings}

This work was funded by the promoters of the CISA: Camões-Institute for Cooperation and Language, Calouste Gulbenkian Foundation, Bengo Provincial Government and the Ministry of Health of Angola. The funders had no role in study design, data collection and analysis, decision to publish, or preparation of the manuscript.

\section{Availability of data and materials}

Data are available from the CISA Institutional Data Access / Ethics Committee for researchers who meet the criteria for access to confidential data.

\section{Authors' contributions}

ER: Participated throughout the entire data collection process, supervised the implementation of the VA system and data collection, contributed to both data analysis and interpretation, drafted the initial manuscript, critically reviewed the manuscript and approved the final submitted manuscript. DC: Contributed to both data analysis and interpretation, drafted the initial manuscript, critically reviewed the manuscript and approved the final submitted manuscript. LT: Participated in data collection and analysis, critically reviewed the manuscript and approved the final submitted manuscript. APR: Conceptualized and designed the study, supervised the implementation of the VA system and data collection, drafted the initial manuscript, contributed to analysis and interpretation, critically reviewed the manuscript and approved the final submitted manuscript. JV: Conceptualized and designed the study, supervised the implementation of the VA system and data collection, contributed to analysis and interpretation, critically reviewed the manuscript and approved the final submitted manuscript. SVN: Conceptualized and designed the study, coordinated and supervised the implementation of the VA system and data collection, contributed to analysis and interpretation, critically reviewed the manuscript and approved the final submitted manuscript. MB: Contributed to analysis and interpretation, coordinated the VA system and data collection, critically reviewed the manuscript and approved the final submitted manuscript.

\section{Competing interests}

The authors declare that they have no competing interests.

\section{Consent for publication}

Not applicable.

\section{Ethics approval and consent to participate}

In the Dande HDSS, we sought verbal consent from household heads or any other adult member of the household, to update the health and demographic information of household members every 6 months. Verbal autopsies for the reported deaths were included as operations of the Dande HDSS.

As a health and demographic surveillance system with frequent visits to households to update demographic information, verbal consent was deemed appropriate for monitoring of the demographic events which requires routine frequent visits to households. In the case of Verbal Autopsy, informed written consent (signed or fingerprinted in case of illiteracy) was required before the conduct of each interview. The information given out to respondents during the consent process was documented and fieldworkers have been adequately trained to administer the consent. The consent, informed about a) the aim of the verbal autopsy system; b) the content of the participation of the caregiver (to answer to an interview, giving information about the death of her/his relative); c) the voluntary feature of participation and d) about the confidentiality of the information. Consent forms were kept safe, strictly confidential and separated from questionnaires. The scientific committee of CISA, composed of several biomedical scientists, epidemiology and public health experts and local health governmental authorities (Provincial Health Governor, Provincial Hospital Director) act as an 
institutional review board (IRB) for all studies and revised the procedures of the HDSS and VA systems. The IRB approved the consent process and also asked for the advice of the Ministry of Health and of Provincial Health Governor. According to their recommendations, the forms of the HDSS and VA were approved and registered in the National Statistics Institute of Angola (INEA) with the number 0019 (Document 746/440/DG/INE/09).

\section{Author details}

${ }^{1}$ Health Research Centre of Angola (CISA), Caxito, Bengo, Angola. ${ }^{2}$ EPIUnit-Institute of Public Health, University of Porto (ISPUP), Porto, Portugal. ${ }^{3}$ Hospital Geral do Bengo, Caxito, Bengo, Angola. ${ }^{4}$ National Institute of Health Doutor Ricardo Jorge, Lisboa, Portugal. ${ }^{5} \mathrm{CRIA}$, Department of Life Sciences, University of Coimbra, Coimbra, Portugal. ${ }^{6} \mathrm{Global}$ Health and Tropical Medicine, GHTM, Instituto de Higiene e Medicina Tropical, IHMT, Universidade Nova de Lisboa, Lisboa, Portugal. ${ }^{7}$ ANU College of Medicine, Biology and Environment, The Australian National University, Camberra, Australia. ${ }^{8}$ Lisbon School of Health Technology (ESTeSL), Lisboa, Portugal.

\section{Received: 31 December 2015 Accepted: 23 July 2016}

\section{Published online: 04 August 2016}

\section{References}

1. Fottrell E, Byass P. Verbal autopsy: methods in transition. Epidemiol Rev. 2010;32:38-55.

2. Mudenda SS, et al. Feasibility of using a World Health Organization-standard methodology for Sample Vital Registration with Verbal Autopsy (SAWY) to report leading causes of death in Zambia: results of a pilot in four provinces, 2010. Popul Health Metr. 2011;9:40.

3. Mathers $C D$, et al. Counting the dead and what they died from: an assessment of the global status of cause of death data. Bull World Health Organ. 2005:83(3):171-7.

4. Sacarlal J, et al. A 10 year study of the cause of death in children under 15 years in Manhica, Mozambique. BMC Public Health. 2009:9:67.

5. Melaku YA, et al. Causes of death among adults in northern Ethiopia: evidence from verbal autopsy data in health and demographic surveillance system. PLoS One. 2014;9(9):e106781.

6. African Development Bank. Angola 2011-2015. Country strategy paper \& 2010 Country Portfolio performance Review. 2011.

7. Butcher A, Jabara C, Jones L. Emerging African economies as growing markets for U.S. Exports, in USITEC executive briefings on trade. 2012.

8. Huong DL, et al. Socio-economic status inequality and major causes of death in adults: a 5-year follow-up study in rural Vietnam. Public Health. 2006;120(6):497-504.

9. Misganaw A, Mariam DH, Araya T. Association of socioeconomic and behavioral factors with adult mortality: analysis of data from verbal autopsy in Addis Ababa, Ethiopia. BMC Public Health. 2013:13:634

10. Braveman P, Tarimo E. Social inequalities in health within countries: not only an issue for affluent nations. Soc Sci Med. 2002;54(11):1621-35.

11. World Health Organisation. Estimated deaths by cause, sex and WHO member state, 2012. Department of Health Statistics and Information Systems. Genebra: World Health Organisation; 2014.

12. Ministério da Saúde de Angola. Relatório de Avaliação Nacional do Sistema de Informação Sanitária. In: Gabinete de Estudos PeE, editor. Luanda, Angola: Ministério da Saúde; 2010.

13. e Pinto BA, Alves JG. The causes of death of hospitalized children in Angola. Trop Doct. 2008;38(1):66-7.

14. Ministério da Saúde de Angola. Plano Nacional de Desenvolvimento Sanitário 2012-2015. Angola: Ministério da Saúde; 2012

15. Costa MJA, et al. Setting up a demographic surveillance system in Northen Angola. Afr Popul Stud. 2012;26(2):133-146.

16. Ministério do Planeamento - Instituto Nacional de Estatística. Inquérito Integrado sobre bem-estar da população. Relatório de tabelas. 2011.

17. Ministério da Saúde de Angola. Plano municipal de desenvolvimento sanitário 2013-2017. Dande-Bengo: Ministério da Saúde; 2014.

18. Anker $M B$, et al. A standard verbal autopsy method for investigating causes of death in infants and children in (WHO/CDS/CSR/ISR/99.4). Genebra: World Health Organization; 1999.

19. INDEPTH-Network. Standard verbal autopsy questionnaire. Adapted from WHO/CDS/CSR/ISR/99.4. 2003.
20. Bang AT, Bang RA. Diagnosis of causes of childhood deaths in developing countries by verbal autopsy: suggested criteria. The SEARCH Team. Bull World Health Organ. 1992;70(4):499-507.

21. Mobley CC, et al. Validation study of a verbal autopsy method for causes of childhood mortality in Namibia. J Trop Pediatr. 1996;42(6):365-9.

22. Quigley MA, Armstrong Schellenberg JR, Snow RW. Algorithms for verbal autopsies: a validation study in Kenyan children. Bull World Health Organ. 1996;74(2):147-54

23. World Health Organisation. Mortality: guideline for certification and rules for coding. Geneva: International Statistical Classification of Diseases and Related Health Problems-tenth revision; 1993.

24. Fikree FF, Azam SI, Berendes HW. Time to focus child survival programmes on the newborn: assessment of levels and causes of infant mortality in rural Pakistan. Bull World Health Organ. 2002;80(4):271-6.

25. Mwanyangala MA, et al. Verbal autopsy completion rate and factors associated with undetermined cause of death in a rural resource-poor setting of Tanzania. Popul Health Metr. 2011;9:41.

26. World Health Organisation. International Statistical Classification of Diseases and Related Health Problems, 10th Revision. Geneva: World Health Organisation; 2010.

27. GBD 2013 Mortality and Cause of Death Collaborators, Salomon JA. Global, regional, and national age-sex specific all-cause and cause-specific mortality for 240 causes of death, 1990-2013: a systematic analysis for the Global Burden of Disease Study 2013. Lancet. 2015;385(9963):117-71.

28. Ahmad OE, Boschi-Pinto C, Lopez AD, Murray CJL, Lozano R, Inoue M. Age standardization of rates: a new WHO standard GPE. Discussion Paper Series: No. 31. Geneva: World Health Organization; 2000.

29. van Eijk AM, et al. Causes of deaths using verbal autopsy among adolescents and adults in rural western Kenya. Trop Med Int Health. 2008;13(10):1314-24.

30. Adjuik $M$, et al. Cause-specific mortality rates in sub-Saharan Africa and Bangladesh. Bull World Health Organ. 2006;84(3):181-8

31. Magalhaes RJ, et al. Finding malaria hot-spots in northern Angola: the role of individual, household and environmental factors within a meso-endemic area. Malar J. 2012:11:385.

32. Sousa-Figueiredo JC, et al. Epidemiology of malaria, schistosomiasis, geohelminths, anemia and malnutrition in the context of a demographic surveillance system in northern Angola. PLoS One. 2012;7(4):e33189.

33. Narh-Bana SA, et al. Adult deaths and the future: a cause-specific analysis of adult deaths from a longitudinal study in rural Tanzania 2003-2007. Trop Med Int Health. 2012;17(11):1396-404

34. Leitao J, et al. Comparison of physician-certified verbal autopsy with computer-coded verbal autopsy for cause of death assignment in hospitalized patients in low- and middle-income countries: systematic review. BMC Med. 2014;12:22

35. Becher $\mathrm{H}$, et al. Patterns of malaria: cause-specific and all-cause mortality in a malaria-endemic area of west Africa. Am J Trop Med Hyg. 2008;78(1):106-13.

36. Soleman N, Chandramohan D, Shibuya K. Verbal autopsy: current practices and challenges. Bull World Health Organ. 2006:84(3):239-45.

37. Kaatano $\mathrm{GM}$, et al. Patterns of malaria related mortality based on verbal autopsy in Muleba District, north-western Tanzania. Tanzan J Health Res. 2009:11(4):210-8.

38. Raviglione MC, et al. Tuberculosis and HIV: current status in Africa. Aids. 1997:11(Suppl B):S115-23.

39. Getahun H, et al. HIV infection-associated tuberculosis: the epidemiology and the response. Clin Infect Dis. 2010:50 Suppl 3:S201-7.

40. Ministério da Saúde de Angola. Discurso do Ministro da Saúde na Abertura do $24^{\circ}$ Conselho Consultivo 16-17.Dez. Angola: Ministério da Saúde; 2014.

41. Patton GC, et al. Global patterns of mortality in young people: a systematic analysis of population health data. Lancet. 2009;374(9693):881-92.

42. Sjursen $\mathrm{IH}$. Angola Health Survey: Opportunities to reduce maternal and newborn mortality, I. Hestad, Editor. 2012, CHR MICHELSEN INSTITUTE \& Centro de Estudos e Investigação Científica.

43. Herbst AJ, Mafojane T, Newell ML. Verbal autopsy-based cause-specific mortality trends in rural KwaZulu-Natal, South Africa, 2000-2009. Popul Health Metr. 2011;9:47

44. Pires JE, Sebastiao YV, Langa AJ, Nery SV. Hypertension in Northern Angola: prevalence, associated factors, awareness, treatment and control. BMC Public Health. 2013:13(1):90.

45. Marquez PV, Farrington JL. Sub-Saharan Africa: The Challenge of NonCommunicable Diseases and Road Traffic Injuries. Washington, DC. The World Bank; 2013. 
46. Jahn A, et al. Child mortality in rural Malawi: HIV closes the survival gap between the socio-economic strata. PLoS One. 2010;5(6):e11320.

47. Hanifi SMA, Mahmood SS and Bhuiya A. Cause-specific mortality and socioeconomic status in Chakaria, Bangladesh. Glob Health Action. 2014; $7(25473)$

48. Kahn K, et al. Socio-economic status and child mortality in a rural sub-distric of South Africa. INDEPTH Network. Measuring health equity in small areas: Findings from demographic surveillance sites. Aldershot: Ashgate; 2005. p. 67-86.

49. Ye M, et al. An improved method for physician-certified verbal autopsy reduces the rate of discrepancy: experiences in the Nouna Health and Demographic Surveillance Site (NHDSS), Burkina Faso. Popul Health Metr. 2011;9:34.

50. Fantahun $\mathrm{M}$, et al. Young adult and middle age mortality in Butajira demographic surveillance site, Ethiopia: lifestyle, gender and household economy. BMC Public Health. 2008;8:268.

51. Dennis EF, et al. Subjective social status and maternal health in a low income urban population. Matern Child Health J. 2012;16(4):834-43.

52. Baiden $\mathrm{F}$, et al. Setting international standards for verbal autopsy. Bull World Health Organ. 2007;85(8):570-1.

53. Byass $P$, et al. Comparing verbal autopsy cause of death findings as determined by physician coding and probabilistic modelling: a public health analysis of 54000 deaths in Africa and Asia. J Glob Health. 2015;5(1):010402.

Submit your next manuscript to BioMed Central and we will help you at every step:

- We accept pre-submission inquiries

- Our selector tool helps you to find the most relevant journal

- We provide round the clock customer support

- Convenient online submission

- Thorough peer review

- Inclusion in PubMed and all major indexing services

- Maximum visibility for your research

Submit your manuscript at www.biomedcentral.com/submit
() BioMed Central 